\title{
Tecnologías Digitales para la atención de personas con Discapacidad Intelectual
}

\section{(Digital Technologies to tend people with Intellectual Disability)}

\author{
Tania Molero-Aranda \\ José Luis Lázaro Cantabrana \\ Mireia Vallverdú-González \\ Mercè Gisbert Cervera \\ Universitat Rovira i Virgili, URV (España)
}

DOI: http://dx.doi.org/10.5944/ried.24.1.27509

\section{Cómo referenciar este artículo:}

Molero-Aranda, T., Lázaro, J. L., Vallverdú-González, M., y Gisbert, M. (2021). Tecnologías Digitales para la atención de personas con Discapacidad Intelectual. RIED. Revista Iberoamericana de Educación a Distancia, 24(1), pp. 265-283. doi: http://dx.doi.org/10.5944/ried.24.1.27509

\section{Resumen}

Este artículo centra su interés en el uso de las Tecnologías Digitales (TD) para la atención de las personas con Discapacidad Intelectual en situaciones de emergencia. Vemos que las TD son percibidas como tecnologías de asistencia para este colectivo, favoreciendo, entre otros aspectos, su autonomía personal. En torno a estos tópicos llevamos a cabo esta investigación cuyo propósito es identificar los beneficios y oportunidades que el uso de las TD puede aportar en la intervención con personas con Discapacidad Intelectual y definir las características que estas deben poseer para atenderles de manera adecuada en situaciones de emergencia. El diseño de investigación es una revisión sistemática, sustentada en la Declaración PRISMA, usando el periodo de búsqueda de enero de 2014 a enero de 2019. Tras el proceso de filtrado de un total de 205 referencias bibliográficas, se han encontrado 14 estudios que cumplen los criterios de inclusión definidos en el proceso. Se ha realizado un análisis de contenido apoyado en códigos, usando para ello Atlas.ti v.8.4. Entre los principales resultados, destacamos que, a pesar del uso normalizado de dispositivos tecnológicos como teléfonos o relojes inteligentes, y de los avances en la accesibilidad de estos, existen numerosas personas que todavía tienen dificultades para realizar una llamada telefónica para pedir ayuda. Es por ello por lo que debemos pensar en tecnologías de asistencia que no dependan únicamente de la acción que el sujeto con discapacidad deba realizar para dar respuestas a situaciones de emergencia en las que se pueda ver implicado. 
Palabras clave: tecnologías digitales; discapacidad intelectual; emergencia; comunicación móvil; tecnología de asistencia.

\begin{abstract}
This article focuses on the use of Digital Technologies for the care of people with Intellectual Disabilities in. We see that Digital Technologies are perceived as assistive technologies for this group, favoring, among other aspects, their personal autonomy. With regard to these topics, we carry out this research, the purpose of which is to identify the benefits and opportunities that the use of Digital Technologies can bring in the intervention with people with Intellectual Disabilities and define the characteristics that they must possess to adequately serve them in situations of emergency. The research design is a systematic review, based on the PRISMA Declaration, using the search period from January 2014 to January 2019. After the filtering process of a total of 205 bibliographic references, 14 studies have been found that meet the inclusion criteria defined in the process. An analysis of content supported by codes has been carried out, using Atlas.ti v.8.4. Among the main results, we highlight that, despite the standardized use of technological devices such as telephones or smartwatches, and the advances in the accessibility of these, many people still have difficulties making a phone call for help. That is why we must think of assistive technologies that do not depend solely on the action that the disabled person must take to respond to emergencies in which he may be involved.
\end{abstract}

Keywords: digital technology; intellectual disability; Emergency; mobile communication; assistive Technology.

Las necesidades de las personas que presentan algún tipo de dificultad comunicativa son altamente individualizadas y complejas (Vuković et al., 2018). Estas dificultades normalmente van asociadas a algún tipo de discapacidad o trastorno. En este caso, nos centraremos en las personas que presentan Discapacidad Intelectual (DI).

Según recoge la American Psychiatric Association (2013) en el DSM-V, la DI se define como una discapacidad caracterizada por limitaciones significativas en el funcionamiento intelectual y en la conducta adaptativa, repercutiendo en las habilidades sociales y en las actividades diarias, manifiestas en la etapa de desarrollo. La DI también puede ser comórbida a otras discapacidades como puede ser el caso del Trastorno del Espectro Autista (TEA). Ambas discapacidades comparten rasgos característicos en cuanto a las habilidades sociales relacionadas con las conductas verbales y no verbales usadas por otros en situaciones sociales, y en actividades diarias como la planificación del tiempo y el espacio (APA, 2013).

Dadas las limitaciones que estas personas presentan en situaciones cotidianas, consideradas para ellos como rutinas, debemos sopesar las dificultades que estas pueden presentar ante situaciones inesperadas y complejas de comprender y gestionar. Pensamos, por ejemplo, en una emergencia por pérdida o accidente de 
tráfico en las que estas se pueden ver implicadas (Lázaro-Cantabrana, SanromàGiménez, Molero-Aranda, Queralt-Romero, Llop-Hernández, 2019). Ante este tipo de situaciones debemos poder garantizar los derechos de seguridad y bienestar social para todas las personas por igual, independientemente de cuales sean sus capacidades.

Las Tecnologías Digitales (TD), teléfonos inteligentes, tablets, tecnologías llevables (wearables technologies) y ordenadores, se han convertido en potentes dispositivos para la comunicación, pero no solo para ello. El incremento del uso de las TD por personas con discapacidad puede ayudar a fomentar su calidad de vida, así como contribuir en su autonomía y participación social (Simplican, Shivers, Chen y Leader, 2018). En este sentido, el desarrollo exponencial de recursos digitales como las aplicaciones móviles (Apps) (Fundación Telefónica, 2018) también han contribuido a la visibilidad de las problemáticas que estas personas pueden tener para realizar actividades diarias como desplazarse de manera segura por su ciudad (Flores et al., 2018), comunicarse mediante Sistema Aumentativo y Alternativo de la Comunicación (SAAC) a través de pictogramas u otros, pudiendo encontrar en el mercado una gran cantidad de estas con finalidades diversas (Buchholz, Ferm y Holmgren, 2017; Kannan et al., 2014; Owuor et al., 2018; Vuković et al., 2016; Vuković, Car, Pavlisa y Mandić, 2018).

Con el fin de explorar experiencias relacionadas con los tópicos del estudio, se ha realizado una Revisión Sistemática (RS) relacionada con el uso de TD para favorecer la atención de personas con algún tipo de discapacidad, concretamente de las personas con DI, en una situación de emergencia. Su proceso y resultados son objeto de esta publicación.

Con la realización de la RS se pretende dar respuesta a las siguientes preguntas de investigación (PI):

PI1. ¿Cuáles son los beneficios y las oportunidades que el uso de las tecnologías digitales aporta a la intervención con personas con DI?

PI2. ¿Qué características tienen las aplicaciones móviles creadas para favorecer la atención de personas con discapacidad en situaciones de emergencia?

A partir de ellas, los objetivos que planteamos son (1) identificar los beneficios y oportunidades que el uso de TD pueden aportar en la intervención con personas con DI y (2) definir las características que deberían cumplir las tecnologías que resultan adecuadas para atender a las personas con DI en situaciones de emergencia.

\section{MÉTODO}

Para realizar la búsqueda de referencias científicas, y garantizar la validación de las mismas, se han tenido en cuenta diversos criterios partiendo de la Declaración PRISMA (Urrútia y Bonfill, 2010) para el diseño y desarrollo del procedimiento, pero también se han tomado como referencia diferentes estudios sistemáticos realizados recientemente en el ámbito de la investigación educativa (Sanromà-Giménez, 
Molero-Aranda, Lázaro-Cantabrana y Gisbert-Cervera, 2018; Vangrieken, Dochy, Raes y Kyndt, 2015; Vangrieken, Meredith, Packer y Kyndt, 2017). 1):

La presente RS se estructura en tres momentos o etapas bien diferenciadas (tabla

- Etapa 1, búsqueda inicial en la literatura científica relacionada con el campo de estudio a través de palabras clave.

- Etapa 2, momento en el que se criban los artículos encontrados siguiendo los criterios de idoneidad establecidos.

- Etapa 3, análisis cualitativo del contenido de los trabajos resultantes de la etapa anterior con el fin de dar respuesta a las preguntas planteadas al inicio del proceso.

Esta RS se realizó de manera colaborativa, revisando todas las referencias encontradas por varios investigadores de manera individual, con la finalidad de minimizar los posibles sesgos producidos por las opiniones formadas previamente por los investigadores respecto a la temática del estudio. También se estableció el criterio de incluir todos aquellos trabajos en los que aparecieron discrepancias y así poderlos reevaluar en fases posteriores. En los casos en los que se presentaba un desacuerdo de interpretación unánime se llegó al consenso entre los investigadores para incluir o descartar las referencias (Gisbert y Bonfill, 2004).

Tabla 1. Proceso de filtrado de la RS: etapas y fases

\begin{tabular}{|c|c|c|c|c|c|}
\hline Etapa 1 & \multicolumn{4}{|c|}{ Etapa 2} & Etapa 3 \\
\hline \multirow{3}{*}{$\begin{array}{l}\text { Búsqueda } \\
\text { inicial de la } \\
\text { literatura en } \\
\text { bases científicas }\end{array}$} & \multicolumn{4}{|c|}{ Criterios de inclusión y selección de estudios } & \multirow{3}{*}{$\begin{array}{l}\text { Análisis de la } \\
\text { literatura en } \\
\text { profundidad }\end{array}$} \\
\hline & Fase 1 & Fase 2 & Fase 3 & Fase 4 & \\
\hline & $\begin{array}{l}\text { Exclusión de } \\
\text { duplicados }\end{array}$ & $\begin{array}{l}\text { Revisión de } \\
\text { títulos }\end{array}$ & $\begin{array}{l}\text { Revisión de } \\
\text { resúmenes }\end{array}$ & $\begin{array}{l}\text { Localización } \\
\text { y descarga }\end{array}$ & \\
\hline
\end{tabular}

A continuación, se describen con detalle cada una de las etapas y fases de la RS atendiendo a las particularidades de esta investigación:

\section{Etapa 1. Búsqueda inicial de la literatura}

La búsqueda inicial de la literatura se realizó a finales del mes de enero de 2019 tomando como marco temporal los últimos 5 años (de enero de 2014 a enero de 2019, ambos incluidos) en las siguientes bases de datos científicas: ISI Web of Science (WoS), SCOPUS, Education Resources Information Center (ERIC) y Google Schoolar. La combinación de palabras claves utilizadas para la búsqueda de trabajos en las diversas bases de datos fueron: (Disability OR Disorder) AND ("Wearable 
Technology" OR "Mobile Technology" OR “Assistive Technology") AND (Safety OR Emergency). El hecho de acotar la revisión temporalmente persigue extraer las publicaciones más actuales en cuanto al problema de investigación abordado, puesto que es en este periodo cuando las tecnologías móviles y el desarrollo de APPs han evolucionado más, a la vez que se puede garantizar su uso para situaciones de emergencia. Esta evolución se ha constatado a nivel de dispositivos (generalización del uso, autonomía, capacidad de los procesadores para alojar APPs con contenido multimedia, etc.), competencia del ciudadano para utilizar dispositivos móviles y conectividad (a partir del $3 \mathrm{G}$ y $4 \mathrm{G}$ ).

Los resultados obtenidos fueron listados en una hoja de cálculo colaborativa atendiendo a los siguientes indicadores: título, autores, año de publicación, DOI, resumen y base de datos científica fuente.

\section{Etapa 2. Criterios de inclusión y selección de estudios}

Tal como muestra la figura 1, la Etapa 2 se compone de diversas fases de filtrado, las que permiten filtrar y valorar la idoneidad de las referencias y ayuda a seleccionar aquellos artículos científicos que puedan ser útiles para dar respuesta a las PI planteadas.

A continuación, se detalla el procedimiento o estrategia definida para hacer la selección de los trabajos en cada una de estas fases:

- Fase 1, de exclusión de duplicados. En esta fase se descartaron aquellos artículos científicos que aparecían repetidamente en el listado inicial fruto de la búsqueda de estudios en diversas bases de datos.

- Fase 2, de revisión de títulos. Para poder filtrar los artículos científicos teniendo en cuenta el título fue necesario definir primero un conjunto de criterios de selección e inclusión en relación con los objetivos planteados al inicio. La estrategia seguida en esta fase se rigió por el cumplimiento de los siguientes criterios de inclusión:

- Estudios que desarrollen intervenciones para mejorar la comunicación de las personas con discapacidad en contextos no educativos.

- Estudios que desarrollen intervenciones para mejorar la calidad de vida de las personas con discapacidad.

- Estudios que incorporen el uso de tecnologías o dispositivos móviles como ayuda o asistencia.

- Fase 3, de revisión de resúmenes. Para esta tercera fase, en la que revisamos el contenido de los resúmenes de los artículos seleccionados, definimos criterios más específicos para el filtrado. La estrategia seguida en esta fase consistió en el cumplimiento de los siguientes criterios de inclusión: 
- Estudios que desarrollan intervenciones para mejorar la comunicación de las personas con discapacidad en diversos contextos no educativos, con especial consideración en aquellos con SAAC.

- Estudios que desarrollan intervenciones para mejorar la calidad de vida de las personas con discapacidad en el ámbito de la seguridad y la autonomía personal.

- Estudios que incorporan el uso de dispositivos móviles, con especial consideración en el uso de tabletas y teléfonos inteligentes.

- Fase 4, de localización y descarga: en esta última fase localizamos y descargamos los artículos científicos resultantes de la selección de la fase anterior para su posterior análisis.

\section{Etapa 3. Análisis de la literatura}

El análisis en profundidad de la literatura seleccionada se llevó a cabo a través de un método de análisis cualitativo del contenido de los artículos.

Para este proceso utilizamos el programa ATLAS.ti 8.4, dadas sus características y herramientas para clasificar información mediante códigos o etiquetas. Estos códigos o etiquetas permiten crear de manera ágil relaciones entre el contenido de los documentos analizados, así como explorar posibles patrones entre los estudios analizados.

Esta etapa pasó también por una estrategia secuenciada, en la que realizamos una primera lectura de las referencias localizadas y descargadas en la fase anterior, y empezamos a identificar aquellos fragmentos que tenían relación con la temática del estudio. En esta ocasión no se partió de la utilización de códigos preestablecidos, sino que se fueron añadiendo a partir de la lectura, sin perder de referencia los objetivos y preguntas marcadas.

\section{RESULTADOS:}

A continuación, se presentan los resultados obtenidos en cada una de las etapas de la RS definida.

\section{Etapa 1:}

En esta primera etapa de búsqueda inicial de la literatura obtuvimos un total de 205 referencias, de las cuales 69 fueron encontrados en la base de datos WOS, 114 en SCOPUS, 10 en ERIC y 12 en Google Scholar. 


\section{Etapa 2:}

En la fase 1, ya descartamos 47 artículos que habían resultado duplicados debido a la búsqueda en diferentes bases de datos. La mayoría de las coincidencias se dieron entre las bases de datos WOS y SCOPUS, entre las que se desestimaron más de esta segunda base por la falta de algunos datos como el DOI o el resumen.

Un dato para destacar de esta primera fase es el número creciente de referencias desde 2014 hasta 2018 (figura 1). Teniendo en cuenta que la búsqueda se realizó en enero de 2019 (incluido) es normal el resultado de referencias obtenidas en ese año.

Figura 1. Número de referencias por año resultantes de la primera fase de la Etapa 2

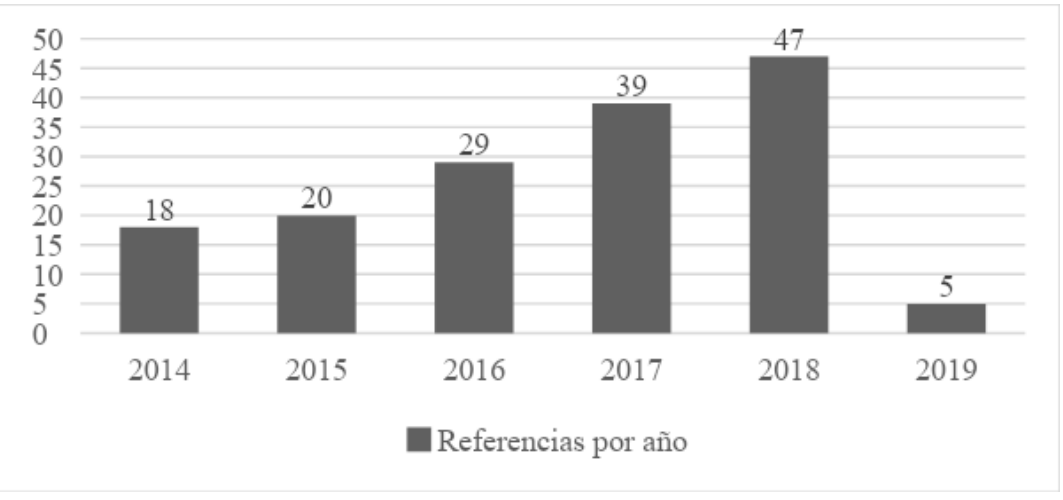

En la fase 2, para la revisión de títulos, incorporamos los criterios de selección detallados en el apartado anterior para valorar la idoneidad de las referencias y descartamos 128, dando como resultado un total de 30 estudios para valorar en la siguiente fase (tabla 2).

Tabla 2. Detalle de referencias seleccionadas en la fase 1 y 2

\begin{tabular}{|c|c|c|c|c|}
\hline $\begin{array}{c}\text { Bases de datos } \\
\text { científicos }\end{array}$ & $\begin{array}{c}\text { Referencias } \\
\text { localizadas }\end{array}$ & $\begin{array}{c}\text { Duplicados } \\
\text { descartados } \\
\text { (Fase1) }\end{array}$ & $\begin{array}{c}\text { Descartados } \\
\text { en la revisión } \\
\text { de títulos } \\
\text { (Fase 2) }\end{array}$ & Seleccionadas \\
\hline WOS & 69 & 8 & 45 & 16 \\
\hline SCOPUS & 114 & 35 & 67 & 12 \\
\hline ERIC & 10 & 0 & 9 & 1 \\
\hline $\begin{array}{c}\text { Google } \\
\text { Scholar }\end{array}$ & 12 & 4 & 7 & 1 \\
\hline Total & 205 & 47 & 128 & $\mathbf{3 0}$ \\
\hline
\end{tabular}


En la fase 3, utilizamos los criterios de selección detallados en el apartado anterior, siendo estos más restrictivos que los utilizados en la fase anterior. En esta fase, con la revisión de los resúmenes de los artículos se pueden empezar a identificar los estudios que realmente tienen relación con los objetivos y criterios establecidos para este proceso. Destacamos el descarte del $40 \%$ de las referencias fruto de la fase anterior, las que pasan a la cuarta y última fase de esta etapa (tabla 3).

Tabla 3. Detalle de referencias seleccionadas en la fase de revisión de resúmenes.

\begin{tabular}{|c|c|c|c|}
\hline $\begin{array}{c}\text { Bases de datos } \\
\text { científicos }\end{array}$ & Referencias fase 2 & Descartados & Seleccionados \\
\hline WOS & 16 & 5 & 11 \\
\hline SCOPUS & 12 & 6 & 6 \\
\hline ERIC & 1 & 1 & 0 \\
\hline Google Scholar & 1 & 0 & 1 \\
\hline Total & 30 & 12 & $\mathbf{1 8}$ \\
\hline
\end{tabular}

Cabe destacar que todos los artículos seleccionados en esta fase responden al criterio de ser estudios que desarrollan intervenciones para mejorar la calidad de vida de las personas con discapacidad en el ámbito de la seguridad y la autonomía personal, haciendo hincapié en el ámbito de la autonomía personal.

En la fase 4, de localización y descarga de los artículos seleccionados, obtuvimos un total de 15 trabajos, los que se introdujeron en el programa ATLAS.ti 8.4 para su posterior análisis de contenido en la Etapa 3.

\section{Etapa 3:}

Después de realizar una primera lectura de todas las referencias encontradas, identificamos un artículo que no respondía ni a los criterios de selección de las fases 2 y 3 de la Etapa 2 ni nos aportan información relevante para alcanzar los objetivos planteados (McSweeney-Feld, 2017). También se identificaron dos pares de artículos que trataban sobre el mismo estudio, pero aportando un punto de vista distinto o aludiendo a los diferentes objetivos de dichas investigaciones (Buchholz, Ferm y Holmgren, 2017; Buchholz, Ferm y Holmgren, 2018; Vuković et al., 2016; Vuković et al., 2018).

El siguiente diagrama de flujo (figura 2) muestra un resumen del proceso de revisión de las referencias a lo largo de toda la RS: 
Figura 2: Esquema del proceso de filtrado de las Etapas y Fases con los resultados obtenidos.

\section{Etapa 1:}

Búsqueda inicial, periodo: 01/2014-2019

$\mathrm{N}=205$

69 WOS. 114 SCOPUS, 10 ERIC, Google Scholar 12



Así pues, se realizó el análisis de un total de 14 publicaciones de tres tipos, 11 de ellas son artículos de revistas, 2 son comunicaciones en congresos y una es un capítulo de libro. Las diferentes producciones científicas analizadas en esta última etapa se presentan ordenadas cronológicamente en la siguiente tabla (tabla 4): 
Tabla 4. Publicaciones analizadas y sus tipos ordenadas cronológicamente

\begin{tabular}{|r|l|l|}
\hline \multicolumn{1}{|c|}{ No $^{\prime}$} & \multicolumn{1}{|c|}{ Autores y año } & \multicolumn{1}{|c|}{ Tipo de publicación } \\
\hline $\mathbf{1}$ & Kannan et al. (2014) & Capítulo de libro \\
\hline $\mathbf{2}$ & Finlayson, Jackson, Mantry, Morrison y Cooper (2015) & Artículo de revista \\
\hline $\mathbf{3}$ & Stough (2015) & Artículo de revista \\
\hline $\mathbf{4}$ & Vuković et al. (2016) & Comunicación en congreso \\
\hline $\mathbf{5}$ & Boyce, Smither, Fisher y Hancock (2017) & Artículo de revista \\
\hline $\mathbf{6}$ & Buchholz, Ferm y Holmgren (2017) & Artículo de revista \\
\hline 7 & Darcy, Green y Maxwell (2017) & Artículo de revista \\
\hline $\mathbf{8}$ & Williamson, Aplin, de Jonge y Goyne (2017) & Artículo de revista \\
\hline $\mathbf{9}$ & Buchholz, Ferm y Holmgren (2018) & Artículo de revista \\
\hline $\mathbf{1 0}$ & Flores et al. (2018) & Comunicación en congreso \\
\hline $\mathbf{1 1}$ & Owuor et al. (2018) & Artículo de revista \\
\hline $\mathbf{1 2}$ & Simplican, Shivers, Chen y Leader (2018) & Artículo de revista \\
\hline $\mathbf{1 3}$ & Vuković, Car, Pavlisa y Mandić, 2018 & Artículo de revista \\
\hline $\mathbf{1 4}$ & Bryant, Brunner y Hemsley (2019) & Artículo de revista \\
\hline
\end{tabular}

Un aspecto que nos pareció también destacable de esta primera exploración de los artículos, y una vez seleccionados aquellos que forman parte de la muestra a analizar, fue el año de publicación, ya que tiene especial relación con la observación que hicimos en la primera etapa de esta RS: los artículos seleccionados tienden a aumentar año a año (ver figura 3).

Figura 3. Comparación entre las referencias localizadas al inicio y las analizadas en la Etapa 3.

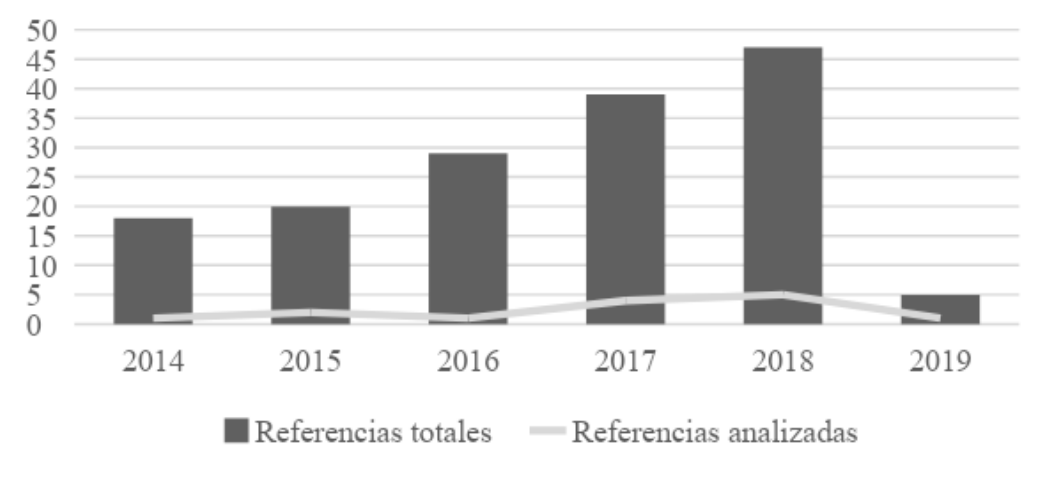


Esta primera lectura también permite la creación de códigos, los que se asocian a diversas citas de los trabajos analizados que versan sobre el concepto en cuestión. En la tabla 5, se detallan los códigos utilizados, así como la frecuencia de la utilización de estos.

Tabla 5. Relación de códigos utilizados para el análisis de contenido con los objetivos propuestos

\begin{tabular}{|c|c|c|}
\hline Objetivo & Código & Frecuencia \\
\hline $\mathbf{1}$ &  & $\begin{array}{c}38 \\
45 \\
8 \\
11\end{array}$ \\
\hline 2 & $\begin{array}{ll}\text { - } & \text { Discapacidad } \\
\text { - DI } \\
\text { - TEA } \\
\text { - Comunicación } \\
\text { - SAAC } \\
\text { - Seguridad / Situación de emergencia descrita }\end{array}$ & $\begin{array}{c}23 \\
4 \\
1 \\
25 \\
12 \\
23\end{array}$ \\
\hline
\end{tabular}

Una vez analizadas en profundidad todas las referencias podemos identificar numerosos beneficios y oportunidades que el uso de las TD pueden aportar en la intervención con personas con diversas discapacidades, pero en especial con las personas con DI. Estos van estrechamente relacionados con la promoción de la autonomía personaly, aunque en segundo plano, también se relacionan con garantizar la seguridad de estos individuos en su independencia (Bryant, Brunner y Hemsley, 2019; Buchholz, Ferm y Holmgren, 2017; Darcy, Green y Maxwell, 2017; Flores et al., 2018; Kannan et al., 2014; Vuković et al., 2016; Vuković et al., 2018; Williamson, Aplin, de Jonge y Goyne, 2017). Entre los beneficios encontramos también que las TD pueden mejorar las relaciones sociales, la comunicación, la autodeterminación, la independencia, la participación y la calidad de vida en general, aunque la falta de adaptabilidad de algunas de estas tecnologías pueden ser una barrera en algunos casos (Buchholz, Ferm y Holmgren, 2017; Darcy, Green y Maxwell, 2017).

Las TD utilizadas en las diversas intervenciones van desde tabletas y teléfonos con sus respectivas Apps (Buchholz, Ferm y Holmgren, 2017; Flores et al., 2018), las que además se pueden conectar a relojes inteligentes o smartwatches (Vuković et al., 2016; Vuković et al., 2018), hasta el uso de la Realidad Virtual (RV) (Bryant, Brunner y Hemsley, 2019). Otra de las tecnologías que se pueden integrar o no a las anteriores es el uso de GPS (Global Positioning System) para, por ejemplo, poder controlar los desplazamientos de las personas con DI, o indicarles de manera accesible el camino para llegar a sus lugares de interés utilizando el transporte público (Flores et al., 2018). 
Algunos de los estudios, pese a no ser estrictamente dirigidos a personas con DI, orientan su utilización al colectivo en caso de ser necesario. Nos referimos al estudio de Kannan et al. (2014) que, centrándose en el uso de una App de navegación por interiores desconocidos para personas con deficiencias visuales, promueven su uso por personas con DI por incorporar estímulos sensoriales que pueden alertar al usuario de algunos peligros en el interior mostrando los recorridos de evacuación en su dispositivo móvil.

De esta manera se puede ver como los dispositivos móviles y las tecnologías llevables se han convertido en potentes tecnologías de asistencia (AT, de sus siglas en inglés assistive technology) para muchas personas con discapacidad (Vuković et al., 2016).

En cuanto a las AT pueden referirse al uso de varios recursos tecnológicos para apoyar a las personas con diferentes discapacidades para obtener beneficios sociales y de comportamiento, y para reducir el impacto negativo de sus discapacidades en su bienestar y participación comunitaria favoreciendo su inclusión (Owuor et al., 2018; Williamson et al., 2017). Las AT también pueden ser estrategias, servicios y prácticas cuyo objetivo principal es mejorar la calidad de vida de las personas con discapacidad.

Según Hersh and Johnson (2008), las AT son tecnologías, equipos, dispositivos, aparatos, servicios, sistemas, procesos y modificaciones ambientales utilizados por las personas con discapacidad o de edad avanzada para superar las barreras sociales, de infraestructura y de otro tipo que impiden la independencia, la plena participación en la sociedad y la realización de actividades de manera fácil y segura (Boyce, Smither, Fisher y Hancock, 2017). Podríamos decir que las AT recogen cualquier elemento o sistema que se use para "aumentar, mantener o mejorar las capacidades funcionales” de una persona con discapacidad (Simplican et al., 2018).

Sin embargo, la Organización Internacional de Normalización con la norma ISO 9999:2016, como punto de referencia para la clasificación de las AT utilizada en muchos sistemas internacionales como la Red Europea de Información sobre Tecnología Asistencial (EASTIN), define como AT tanto las tecnologías convencionales como aquellas desarrolladas específicamente para personas con discapacidad. En esta norma quedan incluidas las AT utilizadas directamente por las personas con discapacidad, así como las que requieren de la asistencia de otras personas para su utilización. Por otro lado, quedan excluidos artículos utilizados para la instalación de AT, medicamentos, productos e instrumentos de asistencia utilizados exclusivamente por profesionales de la salud o soluciones no técnicas, como asistencia personal, perros guía o lectura de labios entre otros.

Así pues, con AT no nos referimos solo a TD, también encontramos estudios (Boyce et al., 2017) en los que el uso de sillas de transporte y evacuación son entendidos como AT, puesto que tienen la finalidad de facilitar y favorecer las tareas de rescate a los cuidadores de personas con DI en situaciones de emergencia. 
En cuanto a las características que las TD poseen o deben poseer para ser adecuadas para atender a las personas con DI en situaciones de emergencia, cabe destacar que todas las intervenciones analizadas (Bryant, Brunner y Hemsley, 2019; Buchholz, Ferm y Holmgren, 2017); Darcy, Green y Maxwell, 2017; Flores et al., 2018; Vuković et al., 2016; Vuković et al., 2018; Williamson et al., 2017) parten de una previa acción de las personas con DI para ser atendidas. Siguiendo esta premisa, es fácil pensar que las TD son accesibles para su uso por este colectivo, pero existe una falta de conocimiento sobre las necesidades de estos usuarios. Es por ello por lo que a las conclusiones que llegan algunos de los estudios (Buchholz, Ferm y Holmgren, 2017; Darcy, Green y Maxwell, 2017; Simplican et al., 2018) es precisamente a la necesidad de involucrar a las personas usuarias en los procesos de investigación para el desarrollo de tecnologías móviles. Argumentan que las personas con discapacidades comunicativas y cognitivas no participan en investigaciones en la misma medida que otras personas, por lo que no se puede esperar que realmente las AT diseñadas respondan o se basen en las opiniones y necesidades del grupo objetivo.

A pesar de ello, algunas de las características que se pueden recoger entre las diversas referencias analizadas son, por ejemplo, el uso de mensajes mediante símbolos e imágenes pictográficas o la incorporación de la capacidad, a los dispositivos móviles, de poder transformar los mensajes de texto en pictogramas (Buchholz, Ferm y Holmgren, 2017) como SAAC. Algunas de estas ya están presentes en Apps que ayudan a los usuarios en tareas personales, vocacionales y educativas (Darcy, Green y Maxwell, 2017).

Otro aspecto para destacar, que los usuarios suelen ver como una limitación, es el hecho de que las aplicaciones diseñadas sean específicas para una tarea y no tengan un carácter flexible en cuanto a poder incorporar algunas de sus características útiles a otras acciones básicas de los dispositivos móviles como puede ser el envío de un mensaje o la realización de una llamada telefónica (Buchholz, Ferm y Holmgren, 2017). En este mismo sentido también se hace hincapié en las actualizaciones de las Apps, puesto que pueden convertirse en un gran problema para la accesibilidad de esta, haciendo que los usuarios con DI deban también "actualizarse" en su uso.

El Informe Mundial sobre Discapacidades (World Health Organization and World Bank, 2011) emitió varias recomendaciones específicas sobre cómo hacer que los entornos sean resilientes durante situaciones de desastre y conflicto (Stough, 2015). Este informe destaca que el uso de la información, siguiendo las indicaciones del diseño universal, desempeña un papel muy importante durante las evacuaciones de emergencia de los edificios, por ejemplo. También se añade que las instrucciones que demos a las personas con DI deben centrar la atención en la información clave para realizar la tarea de manera ordenada y pautada. Las instrucciones paso a paso facilitan la comprensión de la información, puesto que esta se presenta de manera escalable y ordenada. Además, el informe señala la importancia de las AT y las 
comunicaciones durante los desastres, aunque no indican que AT deben utilizarse ni cómo deben ser esas comunicaciones.

En el estudio de Boyce et al. (2017) se recogen las recomendaciones que Clark y Lyons (2010) elaboraron para la presentación de la información sobre los planes de evacuación. Argumentan que en algunas ocasiones las personas que se encuentran en situaciones de emergencia no han estado antes en una situación similar y que por ello es imprescindible que las instrucciones deben:

- Centrar la atención en la información clave para realizar la tarea. En una evacuación, los sistemas sensoriales pueden estar sobrecargados y por ello el hecho de proporcionar buenas señales e indicaciones puede ayudar a reducir la carga cognitiva.

- Usar relaciones que ayuden a las personas a transferir el conocimiento de sus experiencias previas.

- Proporcionar retroalimentación para las acciones correctas e incorrectas para que el error pueda ser eliminado o reconducido.

- Usar gráficos para proporcionar instrucciones paso a paso a los usuarios. Las instrucciones paso a paso proporcionan plegado de andamios, que pueden ayudar con la comprensión.

Otra de las características que pueden poseer las TD para señalizar o notificar avisos en situaciones de emergencia que pueden ser utilizadas en los casos en los que los usuarios lleven un dispositivo móvil o portable pueden ser patrones de vibración, sonidos, gestos y reconocimiento de voz. Estas pueden ser útiles para personas con diversas discapacidades (Kannan et al., 2014).

Otros estudios piden a las autoridades e instituciones recoger evidencias de las intervenciones de atención y asistencia que se dan para ir mejorando las actuaciones con el conocimiento de experiencias previas, ya sean de éxito o fracaso (Boyce et al., 2017; Stough, 2015). También se deja entrever en algunas referencias la necesidad de formación tanto del personal de los servicios de asistencia como (Darcy, Green y Maxwell, 2017; Kannan et al., 2014) de las propias personas con DI y sus familiares más cercanos o sus cuidadores (Buchholz, Ferm y Holmgren, 2017; Kannan et al., 2014; Owuor et al., 2018; Stough, 2015; Vuković et al., 2016; Vuković et al., 2018).

\section{DISCUSIÓN}

Los resultados muestran una escasez de literatura científica respecto al uso de TD para la atención de personas con DI en situaciones de emergencia, aspecto que hace que sea complejo dar respuesta a las preguntas planteadas. Aun así, los hallazgos realizados nos ayudan a trazar las bases para trabajar en la mejora de la realidad de estas personas, tanto ofreciendo respuestas tecnológicas, como trabajando en formación y concienciación al respecto. 
Respecto a la primera PI, hemos podido comprobar que existen numerosos beneficios y oportunidades que el uso de las TD pueden aportar en las intervenciones con personas con diversas discapacidades. Entre estos encontramos la promoción de su autonomía personal, la mejora de las relaciones sociales, la comunicación, la autodeterminación, la independencia, la participación e inclusión social y la calidad de vida en general (Bryant, Brunner y Hemsley, 2019; Buchholz, Ferm y Holmgren, 2017; Darcy, Green y Maxwell, 2017; Flores et al., 2018; Kannan et al., 2014; Vuković et al., 2016; Vuković et al., 2018; Williamson, Aplin, de Jonge y Goyne, 2017).

Las TD más presentes en los trabajos analizados son los dispositivos móviles, como tabletas y teléfonos inteligentes, y los dispositivos llevables, como los relojes inteligentes o smartwatches, a pesar de que su potencial recae principalmente en las aplicaciones que estos dispositivos llevan instaladas (Buchholz, Ferm y Holmgren, 2017; Flores et al., 2018; Vuković et al., 2016; Vuković et al., 2018). Este resultado no nos sorprende dadas las características de estas tecnologías. Su portabilidad y facilidad de uso, además de las adaptaciones a la accesibilidad cognitiva disponible en estos, hace que estas tecnologías sean definidas como AT para las personas con discapacidad, en nuestro caso con DI (Darcy, Green y Maxwell, 2017). Encontramos relevante no haber encontrado estudios relacionados con el uso de asistentes de voz para la atención de personas con discapacidad en situaciones de emergencia en nuestra RS, a pesar de que se conoce su amplia funcionalidad para la mejora de la calidad de vida de este y otros colectivos (Baldauf, Bösch, Frei, Hautle y Jenny, 2018), como las personas mayores, y para funcionalidades relacionadas con la medicina, como el recordatorio de tratamiento farmacológico (Moguel et al., 2019).

A pesar de ello, no todas las personas con DI tienen las mismas capacidades para poder hacer un uso normalizado de estas TD y actividades tan usuales como hacer una llamada telefónica puede ser todo un reto para ellas (Buchholz, Ferm y Holmgren, 2017; Darcy, Green y Maxwell, 2017). En este sentido debemos pensar en las TD como AT utilizadas no solo por las personas con discapacidad, sino también por aquellas tecnologías que requieren de la asistencia de otras personas para su utilización, aspecto muy relevante en nuestro estudio. Es por ello por lo que la definición que más se ajusta a nuestros objetivos de investigación sea la proporcionada por la Organización Internacional de Normalización con la norma ISO 9999:2016.

En cuanto a la segunda PI, en referencia a las características que poseen o deben poseer las TD para atender al colectivo con DI en situaciones de emergencia se hallaron recomendaciones relacionadas con la presentación de la información, donde se puede ver claramente la preferencia hacia indicaciones visuales o sensoriales para facilitar la comprensión de los mensajes (Boyce et al., 2017; Buchholz, Ferm y Holmgren, 2017; Flores et al., 2018; Kannan et al., 2014; Stough, 2015). En este sentido nos ha faltado encontrar información sobre las características que deben poseer las TD para favorecer la atención del colectivo con DI en situaciones de emergencia. 
Si pensamos en el protocolo de actuación que utilizaría una persona neurotípica ante una situación de emergencia, probablemente sería pedir auxilio y/o llamar al 112 para pedir ayuda. Esta es precisamente una de las limitaciones que las personas más afectadas por una DI, puede tener en una situación similar, y así lo demuestran algunos de los estudios analizados (Buchholz, Ferm y Holmgren, 2017; Darcy, Green y Maxwell, 2017; Flores et al., 2018), sobre todo si partimos de que estos muestran soluciones partiendo de la acción de la persona con DI a través de los diversos dispositivos y aplicaciones expuestas. Además, la rigidez e invariabilidad de pensamiento que pueden presentar las personas con DI, así como la carga cognitiva que suelen sufrir en situaciones de estrés como puede ser una situación de emergencia y el desconocimiento a su respuesta o reacción, agravan la atención de estas (Lázaro-Cantabrana et al., 2019).

En este sentido es importante consolidar la evidencia sobre la interacción entre discapacidad intelectual, tecnología de asistencia, vida comunitaria e inclusión social (Vuković et al., 2016) para garantizar su seguridad y bienestar social. Todos los ciudadanos deben poder ser atendidos de manera óptima, pudiendo participar normalmente en la sociedad sin asumir más riesgos.

Por todo ello, debemos pensar en soluciones para aquellas personas con DI que no pueden hacer un uso normalizado de dispositivos y Apps para pedir ayuda en situaciones de emergencia pensando que, además, algunos de ellos presentan graves problemas de comunicación e interacción social. Finalmente, no solo debemos pensar en el tipo de TD a utilizar. La educación y formación sobre el uso de TD, para cualquier finalidad tanto de personas con DI como de los profesionales y familiares que los acompañan, será la que garantice el éxito de estas.

\section{REFERENCIAS}

American Psychiatric Association (APA). (2013). Guía de consulta de los criterios diagnósticos del DSM-5. Arlington, VA: American Psychiatric Publications. https://doi.org/10.1176/appi. books.9780890425657

Baldauf, M., Bösch, R., Frei, C., Hautle, F., y Jenny, M. (2018). Exploring requirements and opportunities of conversational user interfaces for the cognitively impaired. In Proceedings of the 2oth International Conference on human-computer interaction with mobile devices and services adjunct (pp. 119-126). https:// doi.org/10.1145/3236112.3236128

Boyce, M. W., Smither, J. A. A., Fisher, D. O., y Hancock, P. A. (2017). Design of instructions for evacuating disabled adults. Applied ergonomics, 58, 48-58. https:// doi.org/10.1016/j.apergo.2016.05.010

Bryant, L., Brunner, M., y Hemsley, B. (2019). A review of virtual reality technologies in the field of communication disability: implications for practice and research. Disability and Rehabilitation: Assistive Technology, 1-8.

Buchholz, M., Ferm, U., y Holmgren, K. (2017). "That is how I speak nowadays" - experiences of remote communication among persons with communicative and cognitive disabilities. Disability and rehabilitation, 4O(12), 1468-1479. https://doi.org/10.1080/09638288.2017 .1300340 
Buchholz, M., Ferm, U., y Holmgren, K. (2018). Support persons' views on remote communication and social media for people with communicative and cognitive disabilities. Disability and rehabilitation, 1-9. https://doi.org/10.1080/09638288. 2018.1529827

Clark, R. C., y Lyons, C. (2010). Graphics for learning: Proven guidelines for planning, designing, and evaluating visuals in training materials. John Wiley \& Sons.

Darcy, S., Green, J., y Maxwell, H. (2017). I've got a mobile phone too! Hard and soft assistive technology customization and supportive call centres for people with disability. Disability and Rehabilitation: Assistive Technology, 12(4), 341-351. https://doi.org/10.3109/17483107.2016. $\underline{1167260}$

Finlayson, J., Jackson, A., Mantry, D., Morrison, J., y Cooper, S. A. (2015). The provision of aids and adaptations, risk assessments, and incident reporting and recording procedures in relation to injury prevention for adults with intellectual disabilities: cohort study. Journal of intellectual disability research, 59(6), 519-529. https://doi.org/10.1111/jir.12154

Flores, J. Z., Cassard, E., Christ, C., Laayssel, N., Geneviève, G., de Vaucresson, J. B., ... y Radoux, J. P. (2018, July). Assistive Technology App to Help Children and Young People with Intellectual Disabilities to Improve Autonomy for Using Public Transport. In International Conference on Computers Helping People with Special Needs (495-498). Springer, Cham. https://doi.org/10.1007/978-3319-94277-3 76

Fundación Telefónica (2018). Sociedad digital en España 2017. Lectura Plus.

Gisbert, J. P., y Bonfill, X. (2004). ¿Cómo realizar, evaluar y utilizar revisiones sistemáticas y metaanálisis? Gastroenterología y hepatología, 27(3), 129-149. https://doi.org/10.1016/So2105705(03)79110-9
Hersh, M. A., y Johnson, M. A. (2008). On modelling assistive technology systemsPart I: Modelling framework. Technology and disability, 2O(3), 193-215. https:// doi.org/10.3233/TAD-2008-20303

Kannan B., Kothari N., Gnegy C., Gedaway H., Dias M. F., y Dias, M. B. (2014) Localization, Route Planning, and Smartphone Interface for Indoor Navigation. In A. Koubâa y A. Khelil (Eds.), Cooperative Robots and Sensor Networks. Studies in Computational Intelligence, 507, 39-59. Springer, Berlin, Heidelberg. https://doi.org/10.1007/9783-642-39301-3 3

Lázaro-Cantabrana, J. L., Sanromà-Giménez, M., Molero-Aranda, T., Queralt-Romero, M., y Llop-Hernández, M. (2019). Diseño de una aplicación móvil para la seguridad de las personas con trastorno del espectro autista: SOS TEA. Revista de Educación Inclusiva, 12(1), 139-160.

McSweeney-Feld, M. H. (2017). Assistive technology and older adults in disasters: implications for emergency management. Disaster medicine and public health preparedness, 11(1), 135-139. https://doi. org/10.1017/dmp.2016.160

Moguel, E., Azabal, M. J., Flores-Martin, D., Berrocal, J., Garcia-Alonso, J., y Murillo, J. M. (2019). Asistente de voz para el recordatorio de tratamiento farmacológico. Jornadas de Ingeniería del Software y Bases de Datos (JISBD).

Norma Internacional ISO 9999 (2016). Assistive products for persons with disability - Classification and terminology.

Owuor, J., Larkan, F., Kayabu, B., Fitzgerald, G., Sheaf, G., Dinsmore, J., ... y MacLachlan, M. (2018). Does assistive technology contribute to social inclusion for people with intellectual disability? A systematic review protocol. $B M J$ open, 8(2). https://doi.org/10.1136/ bmjopen-2017-017533 
Sanromà-Giménez, M., Molero-Aranda, T., Lázaro-Cantabrana, J. L. y GisbertCervera, M. (2018). Las tecnologías digitales como herramientas de apoyo para la intervención educativa del trastorno del espectro autista: revisión sistemática. EDUTEC 2018. Edicions de la Universitat de Lleida / Asociación EDUTEC, (273-281). https://doi. org/10.21001/edutec.2018

Simplican, S. C., Shivers, C., Chen, J., y Leader, G. (2018). "With a Touch of a Button": Staff perceptions on integrating technology in an Irish service provider for people with intellectual disabilities. Journal of Applied Research in Intellectual Disabilities, 31(1), e130-e139. https://doi.org/10.1111/jar.12350

Stough, L. M. (2015). World Report on Disability, Intellectual Disabilities, and Disaster Preparedness: C osta R ica as a Case Example. Journal of Policy and Practice in Intellectual Disabilities, 12(2), 138-146. https://doi.org/10.1111/ jppi.12116

Urrútia, G., y Bonfill, X. (2010). Declaración PRISMA: una propuesta para mejorar la publicación de revisiones sistemáticas y metaanálisis. Medicina clínica, 135(11), 507-511. $\quad$ https://doi.org/10.1016/j. medcli.2010.01.015

Vangrieken, K., Dochy, F., Raes, E., y Kyndt, E. (2015). Teacher collaboration: A systematic review. Educational Research Review, 15, 17-40. https://doi. org/10.1016/j.edurev.2015.04.002
Vangrieken, K., Meredith, C., Packer, T., y Kyndt, E. (2017). Teacher communities as a context for professional development: A systematic review. Teaching and Teacher Education, 61, 47-59. https://doi. org/10.1016/j.tate.2016.10.001

Vuković, M., Car, Ž., Fertalj, M., Penezić, I., Miklaušić, V., Ivšac, J., ... y Mandić, L. (2016, July). Location-based smartwatch application for people with complex communication needs. In 2016 International Multidisciplinary Conference on Computer and Energy Science (SpliTech), 1-7. https://doi. org/10.1109/SpliTech.2016.7555937

Vuković, M., Car, Ž., Pavlisa, J. I., y Mandić, L. (2018). Smartwatch as an assistive technology: Tracking system for detecting irregular user movement. International Journal of E-Health and Medical Communications (IJEHMC), 9(1), 23-34. https://doi.org/10.4018/ IJEHMC.2018010102

Williamson, B., Aplin, T., de Jonge, D., y Goyne, M. (2017). Tracking down a solution: exploring the acceptability and value of wearable GPS devices for older persons, individuals with a disability and their support persons. Disability and Rehabilitation: Assistive Technology, 12(8), 822-831. https://doi.org/10.1080/ 17483107.2016.1272140

World Health Organization, \& World Bank. (2011). World report on disability. https://www.who.int/publicationsdetail/world-report-on-disability

\section{PERFIL ACADÉMICO Y PROFESIONAL DE LOS AUTORES}

Tania Molero-Aranda. Doctoranda en el programa de Tecnología Educativa de la Universitat Rovira i Virgili. Profesora asociada del Departamento de Pedagogía de la Universitat Rovira i Virgili. Principales líneas de investigación Competencia Digital, Formación del Profesorado e Inclusión Digital. ORCID: 000o-0002-14704549

E-mail: tania.molero@urv.cat 
José Luis Lázaro Cantabrana. Doctor en Tecnología Educativa. Profesor en comisión de servicios del Departamento de Pedagogía de Universitat Rovira i Virgili. Coordinador del Máster Interuniversitario en Tecnología Educativa: e-Learning y Gestión del Conocimiento. Principales líneas de investigación Competencia Digital, Formación del Profesorado e Inclusión Digital. ORCID: 0000-0001-9689-603X E-mail: joseluis.cantabrana@urv.cat

Mireia Vallverdú-González. Graduada en Pedagogía. Colaboradora del Grupo de Investigación Applied Research Group in Education and Technology de la Universitat Rovira i Virgili, E-mail: mireia.vallverdu@urv.cat

Mercè Gisbert Cervera. Doctora en Ciencias de la Educación, Catedrática de la Universitat Rovira i Virgili del Departamento de Pedagogía. Directora del Grupo de Investigación Applied Research Group in Education and Technology de la Universitat Rovira i Virgili y coordinadora del programa de doctorado en Tecnología Educativa de esta misma universidad. ORCID: 0000-0002-8330-1495

E-mail: merce.gisbert@urv.cat

Dirección:

Facultat de Ciències de l'Educació i Psicologia

Universitat Rovira i Virgili

Carretera de Valls s/n

Tarragona (España)

Fecha de recepción del artículo: 14/05/2020

Fecha de aceptación del artículo: 23/07/2020

Fecha de aprobación para maquetación: 31/08/2020 